\title{
The Space Infrared Interferometric Telescope (SPIRIT): the mission design solution space and the art of the possible
}

\author{
David Leisawitz, ${ }^{\mathrm{a}}$ T. Tupper Hyde, ${ }^{\mathrm{a}}$ Stephen A. Rinehart, ${ }^{\mathrm{a}}$ and Michael Weiss ${ }^{\mathrm{a}}$ \\ a NASA’s Goddard Space Flight Center, Greenbelt, MD
}

\begin{abstract}
Although the Space Infrared Interferometric Telescope (SPIRIT) was studied as a candidate NASA Origins Probe mission, the real world presents a broader set of options, pressures, and constraints. Fundamentally, SPIRIT is a far-IR observatory for high-resolution imaging and spectroscopy designed to address a variety of compelling scientific questions. How do planetary systems form from protostellar disks, dousing some planets in water while leaving others dry? Where do planets form, and why are some ice giants while others are rocky? How did high-redshift galaxies form and merge to form the present-day population of galaxies? This paper takes a pragmatic look at the mission design solution space for SPIRIT, presents Probe-class and facility-class mission scenarios, and describes optional design changes. The costs and benefits of various mission design alternatives are roughly evaluated, giving a basis for further study and to serve as guidance to policy makers.
\end{abstract}

Keywords: infrared, submillimeter, interferometry, infrared detectors, cryogenic optics, Origins Probe

\section{INTRODUCTION}

Space mission concept development boils down to resolving the naturally existing tension between the scientific community's thirst for a perfect measuring device and the science policy maker's ability to deliver only limited resources. A scientist is driven by the quest for a deeper understanding of the universe. A policy maker must balance competing requests in light of global priorities and constraints, many of which originate outside the world of science. In addition to external constraints, NASA science policy makers are responsive to scientific community priorities voiced in Decadal Survey reports. The new priorities of the US astrophysics community will be influenced to a greater degree than in the past by practical matters, such as technical readiness, cost, and risk of cost growth.

With a new Decadal Survey in Astrophysics about to begin, we take this opportunity to discuss the SPIRIT mission concept in practical terms. Where does the concept stand in terms of technical readiness? What level of uncertainty should be ascribed to our estimate of the Probe mission's cost? What are the likely impacts of possible design changes in terms of costs and benefits? How is the timing of a SPIRIT mission functionally dependent on programmatic variables and factors, such as the desire to sequence ground and space-based observatories for optimal science pathfinding, or to set the pace of progress toward achieving long-term technical capability objectives; and budgetary constraints and opportunities?

Our aim in writing this paper is to foster enlightened decision-making. Some of the matters under consideration demand subjective reasoning and elicit statements of opinion. Where such opinions are offered, the reader should be aware that these are the authors' personal opinions, not official NASA policy.

Results of the SPIRIT Origins Probe mission concept study were previously reported in a series of papers. The scientific objectives, measurement requirements, and an overview of the design concept are described by Leisawitz et al. (2007). ${ }^{[1]}$ Hyde et al. (2007) ${ }^{[2]}$ summarize system level trades and describe the design concept in greater detail, including the error budget for key design parameters and model-based estimates of the system performance. A paper by Wilson et al. (2007)

${ }^{[3]}$ focuses on the trades that influence optical system design choices, stray light control, metrology, and optical system performance verification. Budinoff et al. (2007) ${ }^{[4]}$ describe the SPIRIT mechanical design and mechanisms and explain how the mechanical design will meet instrument stability, thermal and packaging requirements. DiPirro et al. (2007) ${ }^{[5]}$ present the thermal design concept, thermal modeling results, and the cooling power requirements, and they explain how cryocoolers will be used to meet these requirements. Benford et al. $(2007)^{[6]}$ describe detector requirements, including NEP, pixel count and readout speed, and present the rationale for using small arrays of TES bolometers. Rinehart et al. 
$(2007)^{[7,8]}$ update the status of our development of the wide-field imaging interferometry technique applicable to SPIRIT, and Leisawitz et al. (2007) ${ }^{[9]}$ describe a model of the Wide-field Imaging Interferometry Testbed, a model which can be adapted to simulate interferometric data from SPIRIT.

We begin with a brief description of the SPIRIT Origins Probe mission concept and then proceed to examine the questions raised above.

\section{THE SPIRIT ORIGINS PROBE MISSION CONCEPT}

SPIRIT was designed to accomplish three scientific objectives: (1) revolutionize our understanding of the formation of planetary systems and enable us to "follow the water" as these systems develop; (2) reveal otherwise-undetectable exoplanets; and (3) learn how high-redshift galaxies formed and merged to form the present-day population of galaxies.

To achieve these objectives SPIRIT would provide integral field spectroscopy throughout the wavelength range 25 - 400 $\mu \mathrm{m}$ with sub-arcsecond angular resolution and $\lambda / \Delta \lambda \sim 3000$ spectral resolution in a 1 arcminute instantaneous field of view. Many of the astronomical targets of interest will be resolved for the first time at far-IR wavelengths. SPIRIT's spatially resolved spectra will break model degeneracy and enable a new physical understanding of forming stars and planetary systems, mature planetary systems, and galaxies.

A single scientific instrument gives SPIRIT the powerful combination of measurement capabilities summarized in Table 1. SPIRIT is a Michelson stellar interferometer with a scanning optical delay line for Fourier transform spectroscopy and compensation of external optical path length differences. Following beam combination in the pupil plane, detector arrays multiplex the area coverage, expanding the field of view from the diffraction spot size of the individual light collecting telescopes to the desired arcminute scale. SPIRIT's two telescopes can be moved to sample many interferometric baselines, and therefore to measure spatial structure on all of the angular scales necessary to produce high-quality far-IR images (Figure 1). The image resolution, $0.3(\lambda / 100 \mu \mathrm{m})$ arcsec, is determined by the maximum baseline length, $36 \mathrm{~m}$. The SPIRIT optics are cooled to $4 \mathrm{~K}$ and sensitive detectors are used to attain superlative sensitivity, limited by astrophysical background photon noise.

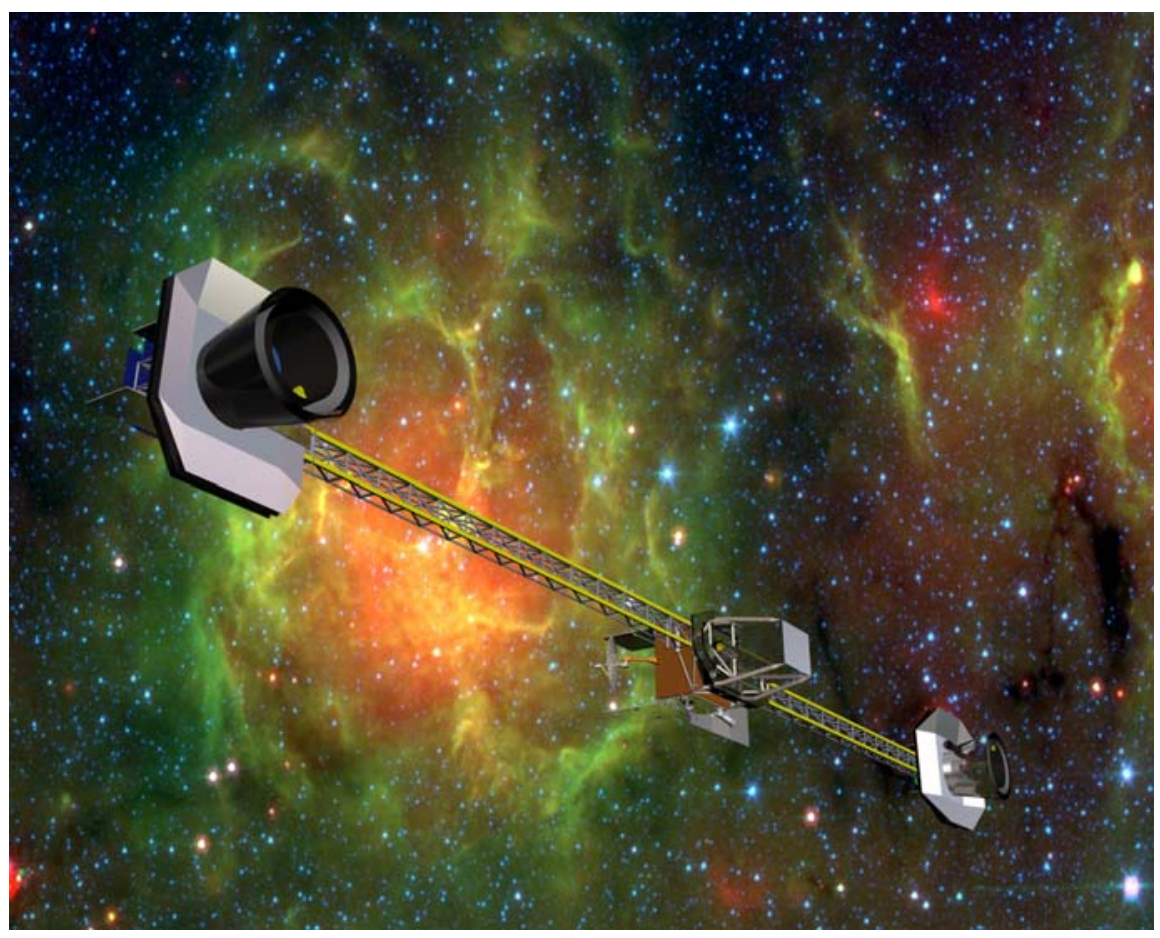

Figure 1. SPIRIT, a far-IR interferometer for imaging and spectroscopy, will revolutionize science requiring high angular resolution. 
Table 1. SPIRIT Origins Probe Design Parameters and Measurement Capabilities

\begin{tabular}{|c|c|}
\hline Wavelength range & 25 to $400 \mu \mathrm{m}$ \\
\hline Instantaneous field of view & 1 arcmin \\
\hline Angular resolution & $0.3(\lambda / 100 \mu \mathrm{m})$ arcsec \\
\hline Spectral resolution $(\lambda / \Delta \lambda)$ & $\sim 3000$ at all wavelengths \\
\hline Point source sensitivity & $\begin{array}{l}3 \times 10^{-18} \mathrm{~W} \mathrm{~m}^{-2} \text { spectral line, } 5 \sigma \text { in } 1 \mathrm{hr} \\
\sim 1 \mu \mathrm{Jy} \text { continuum in "deep field" }\end{array}$ \\
\hline Typical time per target field & $29 \mathrm{hrs}$ \\
\hline Field of regard & $40^{\circ}$ band centered on ecliptic plane \\
\hline Mission life, on station & 3 years (propellant for 5 ) \\
\hline Science data rate (derived) & $5.4 \mathrm{Mb} / \mathrm{s}$ \\
\hline Optics temperature & $4 \mathrm{~K}$ \\
\hline Focal plane temperature & $50 \mathrm{Mk}$ \\
\hline Uncalibrated visibility & 0.90 \\
\hline Phase B start to launch & 72 months (incl. 10 month margin) \\
\hline Telescopes & 2 off-axis, afocal \\
\hline Telescope diameter & $1.0 \mathrm{~m}$ \\
\hline Instrument & $\begin{array}{l}\text { Michelson beam combiner, double } \\
\text { Fourier }\end{array}$ \\
\hline Baseline range & $6 \mathrm{~m}$ to $36 \mathrm{~m}$ \\
\hline Structure & Rigid truss \\
\hline Cryocooling & uprated JWST coolers \\
\hline Sunshield location & Above boom \\
\hline Propulsion system & Hydrazine (monoprop.) \\
\hline ACS type/accuracy & $6 \mathrm{rx}$ wheels, $100 \mathrm{Nm} / \mathrm{s} / 5.0$ arcsec \\
\hline Star trackers & Two on boom \\
\hline Slew rate (peak) & 1 deg per min \\
\hline High gain antenna type & Ka band, two-axis gimbal \\
\hline Ground contacts & $2.0 \mathrm{hr} / 2$ days DSN, Ka band \\
\hline Observatory mass (wet) & 4497 kg (incl. 25\% contingency) \\
\hline Instrument power (EOL) & $\begin{array}{l}1081 \mathrm{~W} \text { for combiner }+1036 \mathrm{~W} \text { per } \\
\begin{array}{l}\text { collector telescope (incl. } 25 \% \\
\text { contingency) }\end{array}\end{array}$ \\
\hline Launch vehicle and fairing & $5 \mathrm{~m}$ diam., Atlas V medium fairing \\
\hline
\end{tabular}




\section{TECHNICAL READINESS}

The technical feasibility of SPIRIT benefits from the current maturity of its technologies, the simplicity and modularity of its design, and the relaxed optical component and design tolerances related to long wavelength observing. Investments made by NASA to develop wavefront sensing and control and cryocooler technology for the James Webb Space Telescope (JWST), and European investments in mechanism technology for Darwin and in far-IR filter technology for Herschel will reduce cost and risk for SPIRIT.

NASA considers a mission ready to pass from the Formulation Phase into the Implementation Phase only after all of the mission-enabling technologies have matured to Technology Readiness Level (TRL) 6. TRL 6 is the point at which a representative model or prototype (sub)system has been functionally demonstrated in a relevant ground or space environment.

Figure 2 shows the SPIRIT mission enabling technologies, gives an estimate of the current state-of-the-art in terms of the TRL for each, and indicates the time required to develop the technology to TRL 6. Many of the SPIRIT mission-enabling technologies are currently at TRL 4 or 5 (Figures 2 and 3). From the time of initial concentrated effort, we estimate that all of the critical technologies can be brought up to TRL 6 in four years.

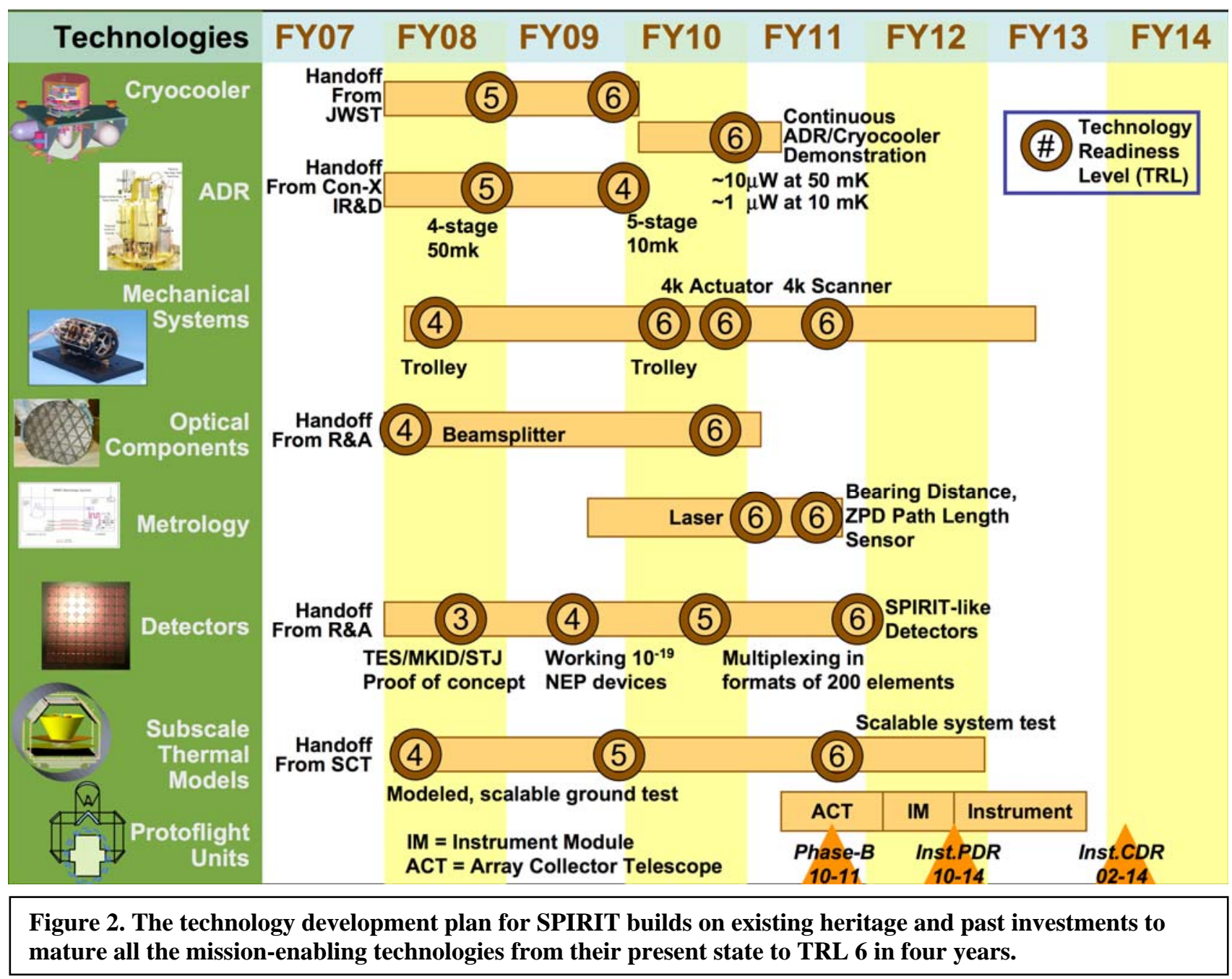

Detectors are the most challenging SPIRIT technology because astrophysicists are the primary customers. Although Michelson interferometers do not need large-format arrays, SPIRIT's detectors must have low NEP and a fast response time. Research suggests that hot-electron TES bolometers will be able to meet the noise requirement and will naturally 
have about the right time constant at $30 \mathrm{mK} \cdot{ }^{[6,10]}$ However, considering the relative immaturity of advanced far-IR detector technology, and as Kinetic Inductance Detectors ${ }^{[11,12,13]}$ and Superconducting Tunnel Junction detectors ${ }^{[14]}$ are potential alternatives to TES bolometers, it seems prudent to invest in parallel competing detector technologies and down-select to the detector type exhibiting the greatest promise. This approach worked for JWST and will guarantee the timely delivery of detectors for SPIRIT.
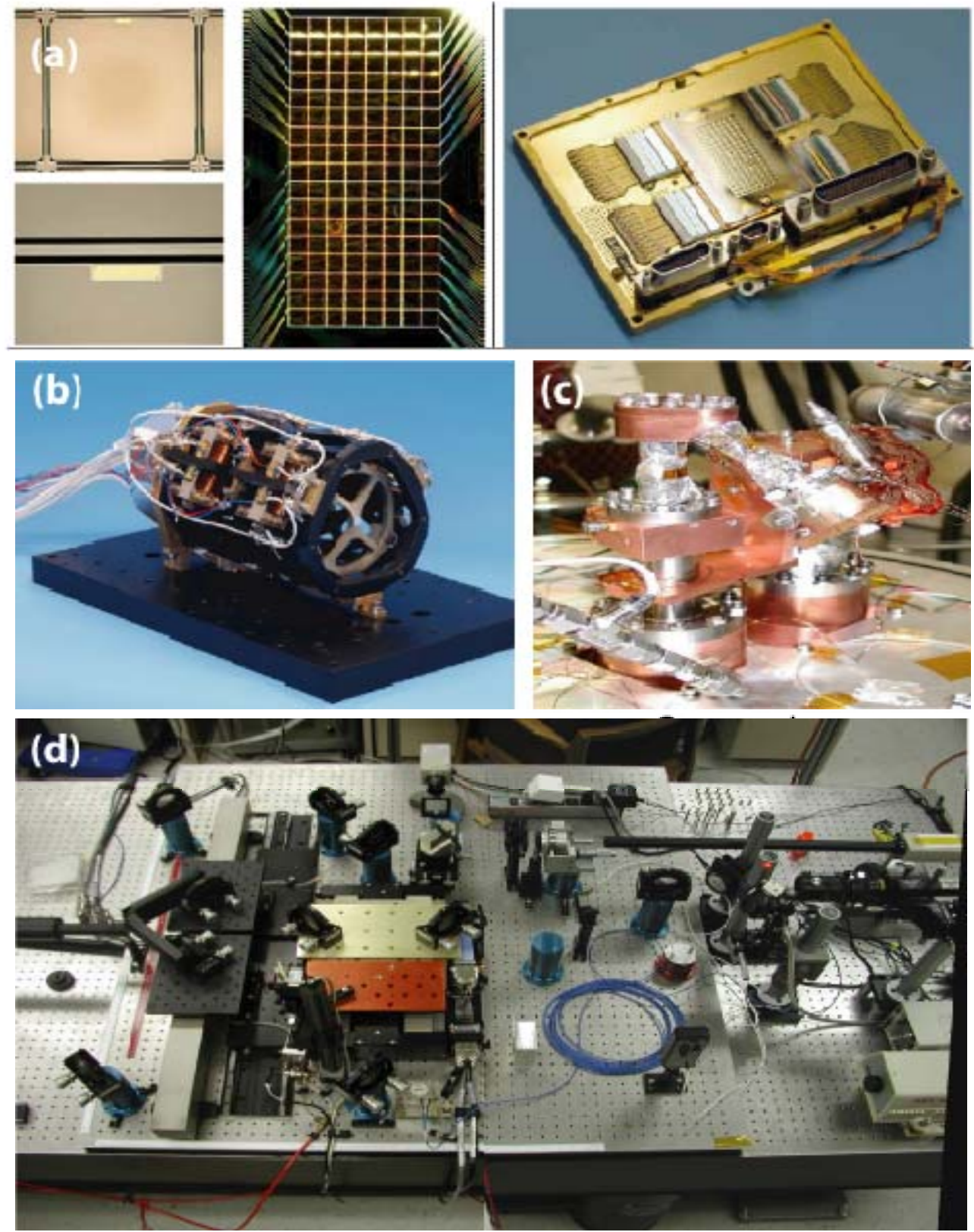

Figure 3. SPIRIT mission-enabling detectors are currently at TRL 3 , and the other key technologies have been developed to Technology Readiness Levels of 4 or 5 . The TES bolometers shown in (a), packaged with readout circuits (photo on the right), have been field demonstrated at the IRAM $30 \mathrm{~m}$ telescope. ${ }^{[6]}$ (b) A cryogenic delay line developed by TNO for the Darwin mission. (c) The cryocooler developed by NorthropGrumman for JWST. (d) The Wide-field Imaging Interferometry Testbed at Goddard Space Flight Center. ${ }^{[8]}$

Thermal system verification is challenging because the thermal balance test will contain warm, room temperature, and cold, $4 \mathrm{~K}$, components. Test chamber non-idealities can dominate the results. For example, the Spitzer thermal balance test showed a measured heat leak of $52 \mathrm{~mW}$ into the instrument when the on-orbit value turned out to be only $6 \mathrm{~mW}$. To improve this situation DiPirro et al. (2007) conducted an experiment using an 18\% scale model of a preliminary SPIRIT telescope design (Figure 4). ${ }^{[5]}$ The test results agreed remarkably well with pre-test thermal model predictions, proving 
out the design and giving us confidence that SPIRIT's telescopes and instrument module can be cooled with JWST-like cryocoolers. A continuously-operating adiabatic demagnetization refrigerator will be capable of providing the required detector cooling.
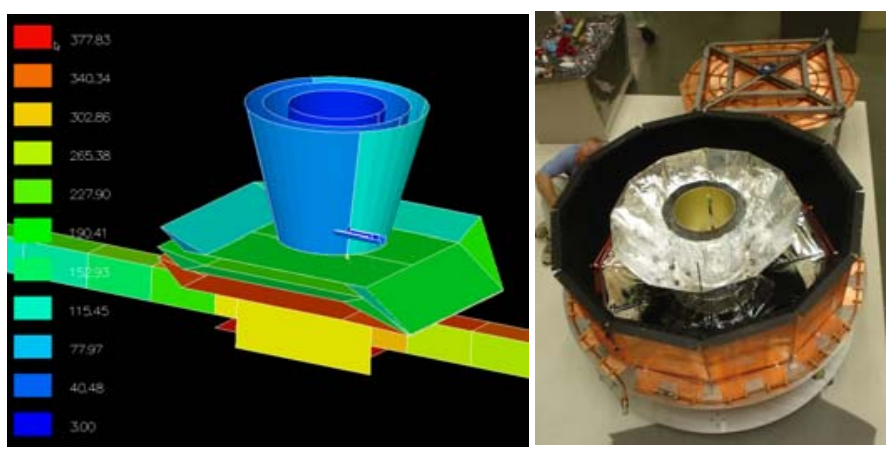

Figure 4. Thermal model results, such as those shown for a single SPIRIT telescope (left) ${ }^{[40]}$ were used to derive heat loads and cryocooler requirements. An early thermal shield design was used in a subscale cryothermal test in a LHe shroud, in which cryocooler stages were simulated with temperature-controlled straps to the shroud (the SCT testbed, right). The test demonstrated the model's accurate predictive capability. ${ }^{[5]}$

Mechanisms similar to those used on SPIRIT have flown successfully on past NASA and ESA missions. For example, cryogenic delay lines have been used in COBE/FIRAS, Cassini/CIRS, and Aura/TES. The Dutch company TNO has developed a precision delay line for the Darwin mission, ${ }^{[15]}$ as well as cryo-mechanisms similar to all the mechanisms SPIRIT requires for beam steering and optical path length control.

In addition to the hardware technologies shown in Figure 2, the practical limitations of the technique of wide-field double Fourier (spatial-spectral) interferometry must be explored and algorithms to enable synthesis of spatial-spectral data cubes from the interferometric data must be demonstrated. The Wide-field Imaging Interferometry Testbed (WIIT) at GSFC was designed to explore practical limitations of the wide field-of-view "double Fourier" technique, which is currently at TRL $4 .^{[7,8,9]}$

\section{COST AND COST UNCERTAINTY}

Several factors moderate the cost of SPIRIT. First, NASA investments made to develop wavefront sensing and control and cryocooler technology for JWST, past investments in cryogenic mechanisms for COBE, Cassini, and Aura, current Research \& Analysis support for far-IR detector technology and wide-field imaging interferometry, and ESA investments in cryogenic mechanisms for Darwin will offset SPIRIT technology development costs relative to our 3-year old estimate. Second, a single scientific instrument on SPIRIT delivers all the required data. Third, the use of cryocoolers instead of cryostats with expendable cryogen significantly reduces launch mass and volume, enabling the use of an Atlas $\mathrm{V}$ launch vehicle with a medium-length fairing.

The aforementioned factors notwithstanding, SPIRIT is expensive enough to necessitate consideration by the general astrophysics community, and ranking in the Decadal Survey. The SPIRIT Origins Probe mission concept study team's grass-roots cost estimate for the mission was $\$ 140 \mathrm{M}$ for technology development, plus $\$ 806 \mathrm{M}$ for Formulation, Implementation and Operation in FY04 US dollars. Apropos a Probe, a bare-bones science operations program lasting only three years was assumed. Reserves based on $43 \%$ of payload cost plus $30 \%$ of flight systems and $10 \%$ of all other mission cost elements were included in the cost estimate. An independent parametric cost model based on a detailed Master Equipment List for the Payload agreed with the team's grass-roots Payload cost estimate to within 20\%, providing a measure of uncertainty. A cost uncertainty factor of, say, $25 \%$ could be applied to the estimated mission cost to derive a fairly robust estimate. After substituting a modern price for the launch vehicle, multiplying by 1.25 to allow for cost uncertainty, and adjusting for inflation, the total lifecycle cost of the mission should be about \$1.3B in FY08.

This is the estimated baseline current-year cost of the mission described in Section 2, and below we will discuss alternative mission design options and their impacts on cost and cost/benefit. 


\section{EXCURSIONS INTO THE MISSION DESIGN SOLUTION SPACE}

\subsection{Enhanced Science Operations: Costs and Benefits}

The short mission lifetime (3 years extendable to 5), and the skimpy science operations plan assumed for a SPIRIT Probe-class mission seem poorly matched to an observatory with SPIRIT's science enabling capabilities, so our first excursion into mission design solution space will look at the costs and benefits of enhanced science operations.

Changes to the flight system designed to enable a longer mission lifetime, say 5 years extendable to 10 , will have a very small cost impact. The only expendable is hydrazine for orbit maintenance at Sun-Earth L2. A larger hydrazine supply could be accommodated within mass and volume constraints of the assumed Atlas V medium launch vehicle, volume being the more precious of the two commodities in the case of SPIRIT. Mechanism lifetime testing and other risk management strategies would have to satisfy more stringent conditions, but the effect on cost would pale in comparison with the cost increment associated with an augmented science operations program.

The bill for science operations for NASA's Great Observatories typically totals several hundred \$M. Scaling from the cost to develop and archive data products and documentation, support guest observers, review observing proposals, plan observations, and fund research grants for the Spitzer Space Telescope, we estimate that a comparable SPIRIT science operations program would cost about $\$ 300 \mathrm{M}$ (integrated over 5 years of science operation).

The benefit to weigh against the cost of a longer mission with a Great Observatory-like guest observer program is the value of the leverage the entire scientific community would bring to the table. Historically, when the community is given vastly improved measurement capabilities, such as those offered by SPIRIT, a number of paradigm-breaking discoveries are made and a mission's science return is greatly amplified. Such discoveries are newsworthy and inspiring to young explorers and the public.

\subsection{Options for a Probe-class Mission}

Alternatively, the SPIRIT mission could be tailored to answer a few key science questions, such as those listed at the beginning of Section 2. This would necessitate a limited set of measurements. The observations could be planned in advance and readily executed in a three-year period. In this case, a competitively selected science team would guide the science program, publish key results, and produce and quickly release data products, enabling follow-up archival analysis by the general astrophysics community. Such a mission would be far less expensive than the one described in Section 5.1 and might qualify as a Probe-class mission if a Probe is allowed to cost $\sim \$ 0.6 \mathrm{~B}$ plus the cost of technology, plus the launch cost (i.e., total cost in the neighborhood of \$0.9B).

If the aim is to stay within a Probe cost cap, given the cost uncertainty mentioned above, a descope plan is the only effective defense against the potential for cost growth. Descope options include a shorter boom to reduce the mechanism count and simplify deployment, or fixed siderostats instead of movable telescopes to reduce the mechanism count and simplify operation. Changes such as these would degrade measurement capabilities and impact science return. Their effects on the viability of the mission as a Probe will not be known until the parameters of a Probe-class mission are clearly defined and further analysis is done.

\subsection{Options for a Facility-Class Mission}

The two preceding sections suggest starkly different options for the SPIRIT mission, the first of which clearly would not fit the Probe mission class as it has been described to date. In this section we examine the implications of a larger, facility-class SPIRIT mission concept. This proves to be liberating in some ways and constraining in others.

On the constraining side, less frequent launch opportunities will exist for more expensive missions. NASA was able to launch the Great Observatories at intervals of about 8 years, with the exception that Hubble and Compton, a relatively inexpensive Great Observatory, were launched only 1 year apart. Projecting from the JWST launch in 2013, the next likely opportunity to launch a comparably expensive observatory won't come until around 2021, with perhaps another one following in 2029. A SPIRIT facility-class mission, if it is one-third the cost of JWST, say, could happen sooner rather than later. 
On the other hand, once the Probe cost cap is breached, any reasonable cost can be considered, permitting mission designers to optimize benefit/cost over a broader cost range. For SPIRIT, the enhanced science operations program discussed in Section 5.1 would represent the first point of departure from the Probe parameter space. This sets the baseline cost of a SPIRIT facility-class mission at about \$1.6B, i.e., the \$1.3B discussed in Section 4, plus \$0.3B for enhanced science operations.

In the following three sections we consider three potential SPIRIT design modifications, each of which would enhance the observatory's measurement capabilities, but at an additional cost. Any of the three potential modifications would likely expand the launch volume requirement for SPIRIT beyond that of the Atlas V medium fairing dimensions. The usable payload volume could be expanded by about $40 \%$ for an additional launch cost of $\sim \$ 40 \mathrm{M}$. This incremental launch cost would add a "knee" to the cost-benefit curve because even a slight enhancement in measurement capability (relative to that summarized in Table 1) would trigger the need for a larger fairing. On the other hand, once the price is paid for a larger fairing, the mission design solution space changes topographically, and a new optimum design solution could emerge. The most appealing measurement enhancements - greater angular resolution, expanded field of regard, and increased sensitivity - all drive the launch volume requirement for SPIRIT. We examine the possibilities below.

\subsubsection{Structure length and angular resolution}

To a first approximation, the boom that holds SPIRIT's telescopes (see Figure 1) could be expanded by 40\% if a $5 \mathrm{~m}$ "long” fairing were substituted for the $5 \mathrm{~m}$ "medium" fairing adopted in the baseline mission concept. This would improve the angular resolution by $40 \%$ relative to $0.3(\lambda / 100 \mu \mathrm{m})$ arcsec, the resolution quoted in Section 2 . The additional payload cost would probably not be large, and plausibly the incremental cost would be dominated by the additional launch cost. Then the question will be: Is $40 \%$ better angular resolution worth an additional $\sim 40 \mathrm{M}$ ? According to the SPIRIT Design Reference Mission, ${ }^{[1]}$ even a $40 \%$ improvement in resolving power may have substantial scientific benefits, so the answer is "possibly yes."

\subsubsection{Sun shades and field of regard}

As illustrated in Figure 1, individual multi-layer sun shades provide passive cooling to SPIRIT's telescopes and beam combining instrument. The shades were sized to permit access to viewing angles up to 20 deg from the anti-Sun direction, and thus to a +/-20 deg band around the ecliptic plane, or about one-third of the sky during the course of the mission. A sufficient number of high-quality astronomical targets are available within that field of regard (FoR) to accomplish the main scientific objectives of the mission.

However, some very interesting targets lie outside the baseline field of regard, and the benefits and cost of enlarging the FoR merit consideration. Without changing the architecture, the FoR can be expanded a little bit with only minor adverse consequences. (With larger sun shades, the movable telescopes could not approach the beam combiner as closely, leaving a larger hole in the short baseline sampling, and degrading the image quality. Computer simulations are needed to assess the impact on science.) Conceivably, SPIRIT could visit targets in up to half of the sky without a major payload design change. The sun shades could be enlarged and still not require deployment if a larger rocket fairing is used.

An architectural change would be required to give SPIRIT access to the entire sky, and this would have a number of repercussions. To enable access to high ecliptic latitudes, SPIRIT's entire boom would have to be protected by a wraparound multi-layer sun shade, something along the lines of the large JWST shade. The shade would have to be folded during launch and deployed, and it's not obvious that it could be packaged for launch in a "long" fairing without a complicated deployment scheme, so new risks would come into play. Alternatively, perhaps, the shade could be launched separately and docked to the rest of SPIRIT, but this would greatly increase the mission cost and complexity. Further complications are likely to stem from the fact that the boom would be cold, so the telescope transport mechanisms would have to operate at much colder temperatures.

In summary, the field of regard sweet spot seems to lie somewhere to the left of the threshold at which the architectural change becomes necessary, but half-sky access is probably within reach.

\subsubsection{Telescope size and sensitivity}

Cost and volume constraints led to the choice of $1 \mathrm{~m}$ diameter telescopes in the SPIRIT Origins Probe study, but relaxation of those constraints would allow the tradeoffs associated with telescope size to be revisited. As noted in Section 2, an interferometer with a pair of cryogenic $1 \mathrm{~m}$ telescopes will provide very good sensitivity, enabling 
accomplishment of the mission's main science objectives. However, a number of measurements will demand the $1 \mu \mathrm{Jy}$ per resolution element sensitivity available only in a “deep field” exposure. It would be very nice to improve SPIRIT's sensitivity by doubling or even tripling the size of the $1 \mathrm{~m}$ diameter telescopes in the baseline SPIRIT mission concept. Fabrication of primary mirrors in the 1 to $3 \mathrm{~m}$ range meeting far-IR performance specifications is not particularly challenging. However, changing the telescope size would affect a number of design parameters. For example, to preserve the 1 arcmin field of view, the detector array dimensions would have to increase in proportion to the telescope diameter. The cryocoolers would have to be more powerful or increase in number.

Just as $1 \mathrm{~m}$ was chosen initially after trading sensitivity (telescope size) for angular resolution (boom length) to meet the original launch volume constraint, this trade will come into play again. It a glance, it seems unlikely that telescopes as large as $3 \mathrm{~m}$ would be favored, as they would likely drive down the boom length intolerably. The payload cost can be expected to increase significantly if the telescope size is doubled, perhaps by two or three times the incremental cost associated with a larger rocket fairing, but the added cost may not be too high a price to pay for the benefit in science return.

Our experience to date suggests that a combination of enhancements would be desired if SPIRIT were to be developed as a facility-class mission. A "long" $5 \mathrm{~m}$ fairing on an Atlas V launcher would become an attractive alternative to the baselined "medium" length fairing, and the science community would ask for increased field of regard, greater sensitivity, and improved angular resolution. Without an enormous cost penalty the field of regard could cover up to half of the sky, the sensitivity could be improved by up to a factor of 4 , and the angular resolution could be improved by up to $40 \%$, but these measurement capabilities can't all be maximized. To find the optimal design consistent with launch on an Atlas V 5 m "long” vehicle (or equivalent) will require careful analysis. Roughly, the incremental cost of a facilityclass SPIRIT mission, above that of the baseline mission cost discussed in Section 4 , totals $\sim \$ 400 \mathrm{M}$, comprising two components: $\sim \$ 100 \mathrm{M}$ for flight system design enhancements leading to improved measurement capabilities, and $\sim \$ 300 \mathrm{M}$ for an enhanced science operations program. The full lifecycle cost of the facility-class observatory would therefore be approximately $\$ 1.7 \mathrm{~B}$, or about one-third the cost of JWST

\section{PROGRAMMATIC CONSIDERATIONS}

The SPIRIT mission will advance NASA's programmatic goals on a broad frontier. For several reasons SPIRIT is a very practical place for NASA to start on the path toward more technically challenging space interferometry missions, which will ultimately be needed to tackle additional NASA scientific objectives. First, interferometry decreases in difficulty as the observed wavelength increases. While picometer units are used to describe the metrology system requirements for some future interferometry missions, the SPIRIT metrology tolerances are a large fraction of a micrometer because of SPIRIT's long wavelengths. Second, technical challenges will be greater when the required interferometric baseline is so large that free flying light collecting telescopes are needed. The modular SPIRIT design shown in Figure 1 is reminiscent of architectures conceived for future interferometers that rely on formation flying; the SPIRIT telescopes and instrument module carry their own power and cooling systems, much as do the telescopes and instruments on longer baseline space interferometers. Third, path length control is not critical with a long-stroke scanning optical delay line, so "double Fourier" interferometry is largely immune to the technical risks associated with visibility amplitude and nulling interferometry.

Vital to planning the TPF-I/Darwin mission is advance knowledge of the mid-IR brightness and spatial structure in the dust disks surrounding candidate target stars. Sensitive measurements of the thermal dust emission at high angular resolution are needed for this purpose. SPIRIT (with $1 \mathrm{~m}$ telescopes) will have at least the sensitivity to detect a 3 "zodi” disk $\left(1\right.$ zodi $\left.=10^{-6} \mathrm{~L}_{\mathrm{Sun}}\right)$ or to map the distribution of dust in a resolved 100 zodi disk at $10 \mathrm{pc}$ in a 1 -week observation period, and better sensitivity is possible, as noted in Section 5.3.3. Thus, SPIRIT observations could set the stage scientifically for follow-up detection and characterization of terrestrial planets with a mid-IR nulling interferometer.

\section{SUMMARY}

This paper maps SPIRIT onto the real-world landscape as we perceive it at the present time. In light of the scientific community's interests and priorities, as voiced at the May 2008 conference "Far-IR Astronomy from Space: A Community Workshop About the Future," we conclude that a facility-class SPIRIT mission, with measurement capabilities augmented slightly relative to those of the baseline SPIRIT Probe design concept, would enjoy a broad base of support. We have roughly estimated the cost of a facility-class version of SPIRIT and found it to be on the low end of 
the price range of NASA's Great Observatories. A precedent set by the Compton Gamma Ray Observatory suggests that NASA might be able to afford to develop SPIRIT around 2020, even if the 2021 slot nominally available for a strategic mission successor to JWST is given to a different mission.

\section{ACKNOWLEDGMENTS}

NASA's Goddard Space Flight center managed and provided scientific leadership and engineering expertise for the SPIRIT Origins Probe study. In addition to the study participants at Goddard, the SPIRIT team includes representatives from NASA's JPL and MSFC, academia, industry, and international partners.

\section{REFERENCES}

${ }^{[1]}$ Leisawitz, D., Baker, C., Barger, A., Benford, D., Blain, A., Boyle, R., Broderick, R., Budinoff, J., Carpenter, J., Caverly, R., Chen, P., Cooley, S., Cottingham, C., Crooke, J., DiPietro, D., DiPirro, M., Femiano, M., Ferrer, A., Fischer, J., Gardner, J.P., Hallock, L., Harris, K., Hartman, K., Harwit, M., Hillenbrand, L., Hyde, T., Jones, A., Kellogg, J., Kogut, A., Kuchner, M., Lawson, W., Lecha, J., Lecha, M., Mainzer, A., Mannion, J., Martino, A., Mason, P., Mather, J.C., McDonald, G., Mills, R., Mundy, L., Ollendorf, S., Pellicciotti, J., Quinn, D., Rhee, K., Rinehart, S.A., Sauerwine, T., Silverberg, R.F., Smith, T., Stacey, G., Stahl, H.P., Staguhn, J., Tompkins, S., Tveekrem, J., Wall, S., and Wilson, M., "The Space Infrared Interferometric Telescope (SPIRIT): High-resolution imaging and spectroscopy in the far-infrared,” Adv. Sp. Res., 40, 689 (2007), doi:10.1016/j.asr.2007.05.081

${ }^{[2]}$ Hyde, T.T., Leisawitz, D.T., DiPietro, D., and Rinehart, S.A., "System engineering the Space Infrared Interferometric Telescope (SPIRIT),” Proc. SPIE 6687, 66870A1 - 12 (2007).

${ }^{[3]}$ Wilson, M.E., Leisawitz, D.T., Martino, A.J., Rinehart, S.A., Crooke, J.A., Tveekrem, J.L., Budinoff, J.G., Quijada, M.A., and Hyde, T.T., "The Space Infrared Interferometric Telescope (SPIRIT): Optical system design considerations," Proc. SPIE 6687, 66870B1 - 11 (2007).

${ }^{[4]}$ Budinoff, J.G., Leisawitz, D.T., Rinehart, S.A., DiPirro, M.J., Jones, D.L., Hyde, T.T., and Taylor, B., "Mechanical design of the Space Infrared Interferometric Telescope (SPIRIT),” Proc. SPIE 6687, 66870C1 - 11 (2007).

${ }^{[5]}$ DiPirro, M.J., Cottingham, C., Boyle, R., Ollendorf, S., and Leisawitz, D.T., “The SPIRIT thermal system,” Proc. SPIE 6687, 66870D1 - 11 (2007).

${ }^{[6]}$ Benford, D.J., Rinehart, S.A., Hyde, T.T., and Leisawitz, D.T., “Cryogenic far-infrared detectors for the Space Infrared Interferometric Telescope (SPIRIT),” Proc. SPIE 6687, 66870E1 - 12 (2007).

${ }^{[7]}$ Rinehart, S.A., Armstrong, J.T., Frey, B.J., Jung, J., Kirk, J., Leisawitz, D.T., Leviton, D.B., Lyon, R.G., Martino, A.J., Mundy, L.G., Pauls, T.A., and Schurr, S., "Wide-field imaging interferometry: An enabling technique for high angular resolution astronomy,” Proc. SPIE 6687, 66870F1 - 10 (2007).

${ }^{[8]}$ Rinehart, S.A., Leisawitz, D., Frey, B., Lyon, R., Maher, S., and Memarsadeghi, N., "The Wide-Field Imaging Interferometry Testbed (WIIT): Recent Progress and Results,” Proc. SPIE 7010, this volume (2008).

${ }^{[9]}$ Leisawitz, D.T., Martino, A.J., Thompson, A.K., Rinehart, S.A., and Frey, B.J., “An optical model of the Wide-field Imaging Interferometry Testbed,” Proc. SPIE 6687,66870J1 - 9 (2007).

${ }^{[10]}$ Day, P., LeDuc, H., Dowell, C.D., Lee, R.A., Turner, A., and Zmuidzinas, J., “Distributed Antenna-coupled TES for FIR detector arrays,” J. Low Temp. Phys., 151, 477 (2008).

${ }^{[11]}$ Mazin, B.A., Day, P.K., LeDuc, H.G., Vayonakis, A., and Zmuidzinas, J., “Superconducting Kinetic Inductance Photon Detectors,” Proc. SPIE 4849, 283 (2002).

${ }^{[12]}$ Sergeev, A., Karasik, B., Gogidze, I., and Mitin, V., "Ultrasensitive Hot-Electron Kinetic-Inductance Detectors,” in Ninth International Workshop On Low Temperature Detectors, F.S. Porter, D. McCammon, M. Galeazzi \& C.K. Stahle, eds., AIP Conf. Proc. 605, 27 (2002).

${ }^{[13]}$ Baselmans, J., Yates, S.J.C., de Korte, P., Hoevers, H., Barends, R., Hovenier, J.N., Gao, J.R., and Klapwijk, T.M., "Development of high-Q superconducting resonators for use as kinetic inductance detectors," Adv. Sp. Res., 40, 708 (2007). 
${ }^{[14]}$ Schoelkopf, R.J., Moseley, S.H., Stahle, C.M., Wahlgren, P., and Delsing, P., “A Concept for a Submillimeter-Wave Single-Photon Counter,” IEEE Trans. on Appl. Superconductivity, 9, 2935 (1999).

${ }^{[15]}$ van den Dool, T.C., Kamphues, F., Gielesen, W., Dorrepaal, M., Doelman, N., Loix, N., Verschueren, J.P., Kooijman, P.P., Visser, M., Velsink, G., and Fleury, K., "The manufacturing, assembly and acceptance testing of the breadboard cryogenic Optical Delay Line for DARWIN,” Proc. SPIE, 5904, 590415-1 - 11 (2005). 